\title{
An improved model for the damped impact of composite materials applicable to wind turbine blades
}

\author{
Florina-Carmen Ciornei $^{1, *}$ Stelian Alaci ${ }^{1}$, Sorinel-Toderas Siretean ${ }^{1}$ and Mariana-Catalina Ciornei $^{2}$ \\ ${ }^{1}$ Stefan cel Mare University, Suceava, 13 University Str., 720229, Romania \\ ${ }^{2}$ Carol Davila University of Medicine and Pharmacy, Dpt. Clinic 2, 8 Bd. Eroii Sanitari, 050474, Bucharest, Romania
}

\begin{abstract}
Composite materials are used in manufacturing a vast game of products, from usual objects, medical devices, aerospatial parts to military equipment. One of the features considered when a composite material is designed as target aims the energy dissipation with respect to the projectile. The paper presents an improved alternative of a model which is broadly used in the impact of projectile and composite target. The initial model has the weakness that does not present the hysteresis loop. The improvement of the model consists in including a nonlinear damper placed between the projectile and the target, the damping force being simultaneously proportional to the impact velocity and the elastic force. The new model has a hysteresis loop closed in the origin. The proposed model is applied for concrete situations and the results are presented in graphical manner.
\end{abstract}

\section{Introduction}

Composite materials are widely used in developing the products met in everyday life [1] but also in specialized domains, like medical devices [2-5], military equipment or wind electric systems [6]. Theoretically they can be designed for achieving preferred characteristics but as Abrate highlights [7-10] the domain of application is narrowed by the impact behavior. The numerous papers dedicated lately to this matter prove the increasing attention produced by this subject.

A number of papers study the impact at low velocities and the impact strength. Abrate [11] underlines that a first step in understanding the effect consist in the development of a model capable to allow the prediction of time evolution of the impact force and of the reaction of the structure. The analysis of impact behavior emphasizes that any impact model is situated between two limit situations: the first case, when the entire energy of the projectile is transferred to the target, and small deformations are produced that finally lead to crack micro initiators; the second case, when the composite, due to its properties, returns the entire impact energy to the projectile. A typical case for the situations mentioned above are the rotor blades of the wind turbines made of composite materials, Mishnaevsky [12]. A recent paper due to Gaudern [13] proofs the destructive effect produced by the erosion upon the rotor blades of the wind turbine. The statement is demonstrated with probes obtained in wind tunnels. To be remarked that the leading edge is the most affected region of the rotor blade. As known, the wind turbine farms must be placed in regions where minimum impact upon the environment is produced. Onshore, it is expected to view wind turbines working quite remote from popular areas, even in agricultural lands or in desert regions. A characteristic of these regions is the presence of dust particles that will be swept up into motion by the large air masses with velocities up to $13 \mathrm{~m} / \mathrm{sec},[14]$ and thus becoming actual micro-projectiles on the surface of the turbine. The mentioned facts are good reasons for the conception of models capable to estimate the impact force and lastly to allow for optimum design of rotor blades of wind turbines.

\section{The Abrate spring-mass models for the impact of composite structures}

Abrate makes a comprehensive review [11] concerning the representations that study the projectile-composite material impact and identifies several models.

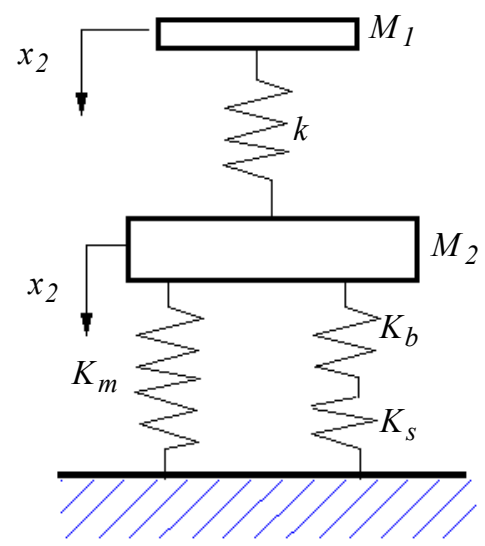

Fig. 1. The spring-mass model studied by Abrate [11]

\footnotetext{
* Corresponding author: florina@fim.usv.ro
} 
Among these the spring-mass models are present. These models have the advantage of being simple and offering precise solutions for certain impact types, frequently met during the tests where small projectiles are used.

A two degree of freedom model is presented in [11] and consists in a deformable structure (2) collided by a projectile of small dimensions (1), as presented in Fig. 1.

The system is modeled using two masses and three springs. The equations describing the motion of the system are:

$$
\left\{\begin{array}{l}
M_{1} \ddot{x}_{1}+P=0 \\
M_{2} \ddot{x}_{2}+K_{b s} x_{2}+K_{m} x_{2}^{3}-P=0
\end{array}\right.
$$

where $M_{1}$ is the mass of the projectile, $K_{b s}$ is the elastic constant of the structure with linear behaviour, $K_{m}$ is the elastic constant corresponding the the nonlinear behaviour of the structure. In equation $1 P$ is the impact force between the structure and the projectile, expressed as a strongly nonlinear dependency on the deformation $x_{2}-x_{1}$.

Next it is assumed that the impact force $P$ presents a Hertzian form. Thus, for the case of centric impact of two spheres, the impact force has the expression:

$$
\begin{gathered}
P=K\left(x_{2}-x_{1}\right)^{\frac{3}{2}} \\
K=\frac{4}{3 \pi}\left(\frac{R_{1} R_{2}}{R_{1}+R_{2}}\right)^{\frac{1}{2}}
\end{gathered}
$$

where $R_{1,2}$ are the radii of curvature of the contacting bodies and $\eta$ is the contact stiffness, defined according to Johnson [15] as:

$$
\eta=\frac{1-v_{1}^{2}}{E_{1}}+\frac{1-v_{2}^{2}}{E_{2}}
$$

In the above relation, $v_{12}$ and $E_{1,2}$ are the Poisson coefficients and the Young moduli, respectively, of the two materials. Next it is considered the impact between a ball-projectile and plane membrane-target. For a complete clamped isotropous circular membrane, the coefficients $K_{m}$ and $K_{b}$ are expressed by the relations:

$$
\begin{gathered}
K_{b}=\frac{4 \pi E h}{3\left(1-v^{2}\right) a^{2}} \\
K_{m}=\frac{(353-191 v) \pi E h}{648\left(1-v^{2}\right) a^{2}}
\end{gathered}
$$

where $h$ is the thickness and $a$ is the radius of the membrane, [16]. To be mentioned that the effective mass of the membrane taking part to the oscillation motion is a quarter of the total mass of it. The equations 1 considered together form a system of nonlinear differential equations ,[17] and solving it requires applying a numerical procedure, [18]. The initial conditions are necessary in order to integrate the system and in the present case they are:

$$
\begin{aligned}
& x_{1}=0 \\
& x_{2}=0 \\
& \dot{x}_{1}=v \\
& \dot{x}_{2}=0
\end{aligned}
$$

The next concrete situation was considered for integrating the system:

$$
\begin{aligned}
& a=0.1 \mathrm{~m}, \\
& h=0.005, \\
& R_{1}=0.02 \mathrm{~m}, \\
& R_{2}=2 \cdot 10^{3} \mathrm{~m}
\end{aligned}
$$

Both the ball and the membrane are made of steel and have the elastic modulus $E_{1,2}=2.1 \cdot 10^{11} \mathrm{~Pa}$. The Runge-Kutta IV method, [18] was applied for integrating the system of equations 1 .

The absolute displacements of the projectile $x_{1}$, of the center of the membrane $x_{2}$ and the relative displacement of the projectile with respect to the membrane are presented in Fig. 2.

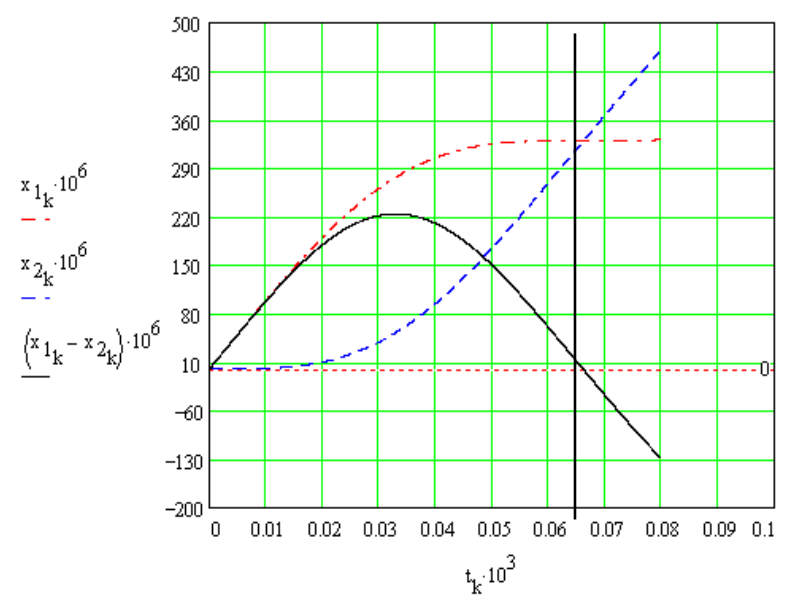

Fig. 2. Absolute deformations of the ball $x_{1}$, of the membrane $x_{2}$, and the relative deformation $\left(x_{1}-x_{2}\right)$

From Fig. 2 it is noticed that at a certain instant, the deformation of the membrane equals the deformation of the ball.

The deformations from Fig. 2 are represented for a longer period in Fig. 3. It can be remarked that both the projectile and the membrane get an oscillatory motion as a result of at least two harmonics and therefore, the relative displacement of the projectile with respect to the target is a quasi sinusoidal oscillation. 
The plot force-relative deformation of the projectile presented in Fig. 4 is of particular attention.

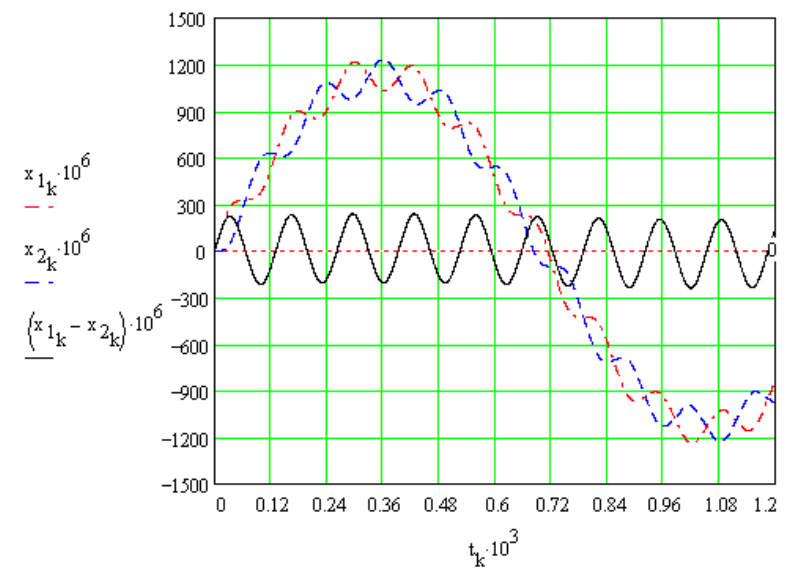

Fig. 3. Absolute deformations (of the ball $x_{1}$, of the membrane $\left.x_{2}\right)$ and relative deformation $\left(x_{1}-x_{2}\right)$ for longer time

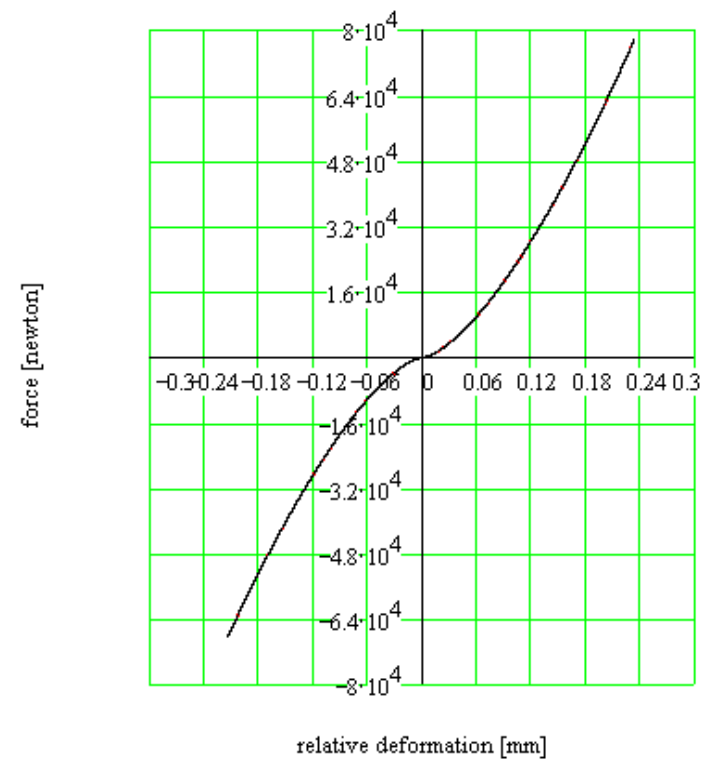

Fig. 4. Force-relative deformation curve for the projectile

From Fig. 4 it can be concluded that the system of differential equation 1 describe the behavior of an undamped system since for a damped action, a closed forcedeformation curve entrapping an area equal to the work of damping should occur.

The fact that the model of a composite material exhibits un-damped behaviour is a major deficiency of the representation.

\section{Proposal of improved model}

In order to improve the model proposed by Abrate, a viscous dashpot (damper) is attached to the model, connected in parallel to the spring between the projectile and the membrane. As it can be observed form Fig. 5, the damping constant was considered variable.

This requirement was applied by Lankarani and Nikravesh [19], and Flores et al. [20]; for modeling an impact phenomenon with viscous damping, they establish the model based on the remark made by Hunt and Crossley [21], who showed that when the viscous damping depends only on the relative velocity the hysteresis loop is open in origin, this meaning that at the end of the impact, the two bodies attract to each other instead of rejecting.

Based on the observations made by Dubowsky and Freudenstein [22-23], Hunt and Crossley [21] showed that for obtaining a loop closed in origin it is necessary that the damping force should be proportional both to relative velocity and to the contact force exerted when no damping is present.

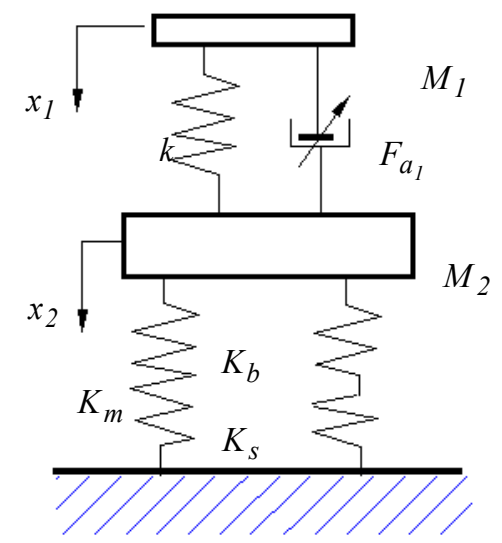

Fig. 5. Proposed model with damper

Founded on this remark, a modified equation of motion, that considers the damping occurring in the ballcomposite contact, is proposed under the following form:

$$
\left\{\begin{aligned}
\ddot{x}_{1} & =\left[-\frac{K}{M_{1}}-\frac{\kappa}{M_{1}}\left(\dot{x}_{1}-\dot{x}_{2}\right)\right]\left|x_{1}-x_{2}\right|^{\alpha} \operatorname{sgn}\left(\dot{x}_{1}-\dot{x}_{2}\right) \\
\ddot{x}_{2} & =\left[-\frac{K_{b}}{M_{1}} x_{2}-\frac{\kappa}{M_{2}} x_{2}^{3}\right]+ \\
& +\left[\frac{K}{M_{1}}+\frac{\kappa}{M_{1}}\left(\dot{x}_{1}-\dot{x}_{2}\right)\right]\left|x_{1}-x_{2}\right|^{\alpha} \operatorname{sgn}\left(\dot{x}_{1}-\dot{x}_{2}\right)
\end{aligned}\right.
$$

In the relations 8 , the parameter $\kappa$ is the characteristic of damping and $\alpha=3 / 2$ is the exponent typical to the Hertzian contact [24-27].

The system of differential equations 8 was integrated numerically for the parameters 7 and for the same initial conditions 6 , for two values of the $\kappa$ constant, that is: $\kappa=10^{9}, \kappa=3 \cdot 10^{9}$ (units $N \cdot s / m^{1+\alpha}$ ).

For thes $3 \mathrm{e}$ two values of the $\kappa$ constant, there are presented comparatively:

- the absolute displacements of the projectile and target and the relative displacement, in Fig. 6;

- $\quad$ the impact forces versus time in Fig. 7;

- $\quad$ the hysteresis loops in Fig. 8. 

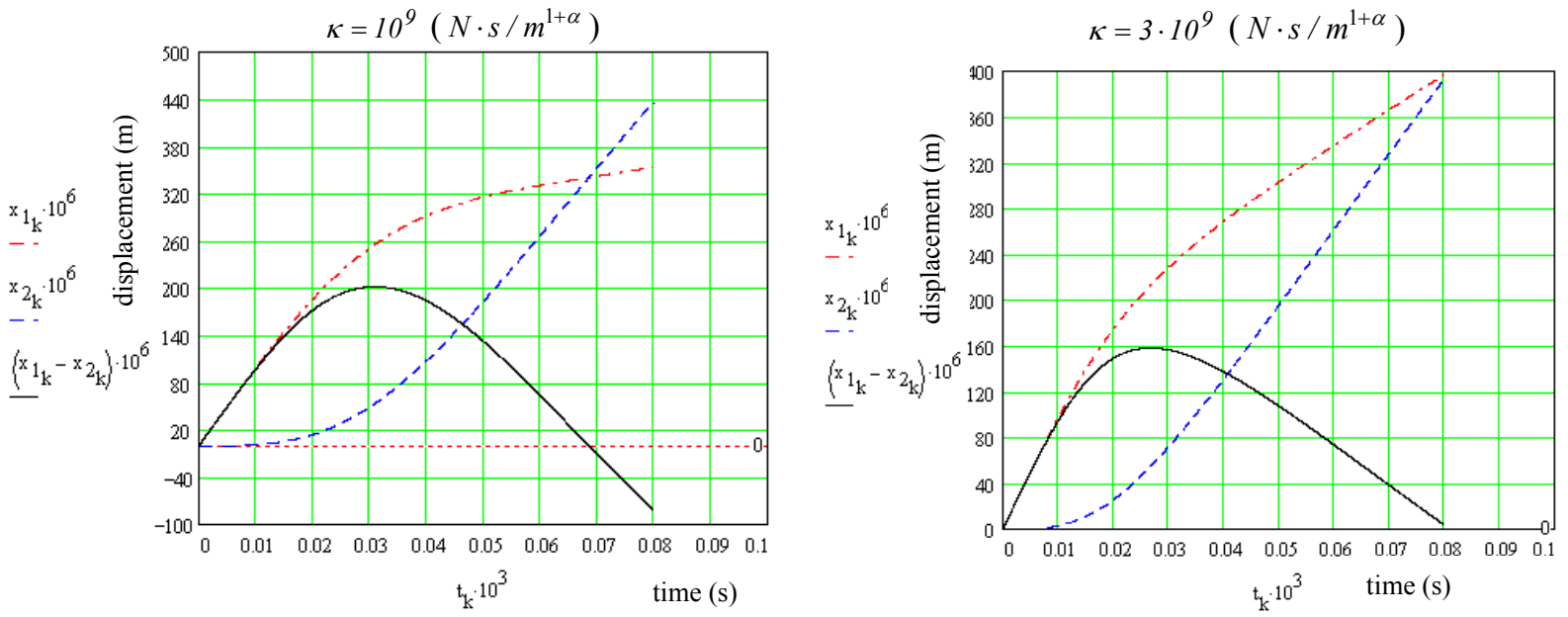

Fig. 6. Displacements of the projectile and target
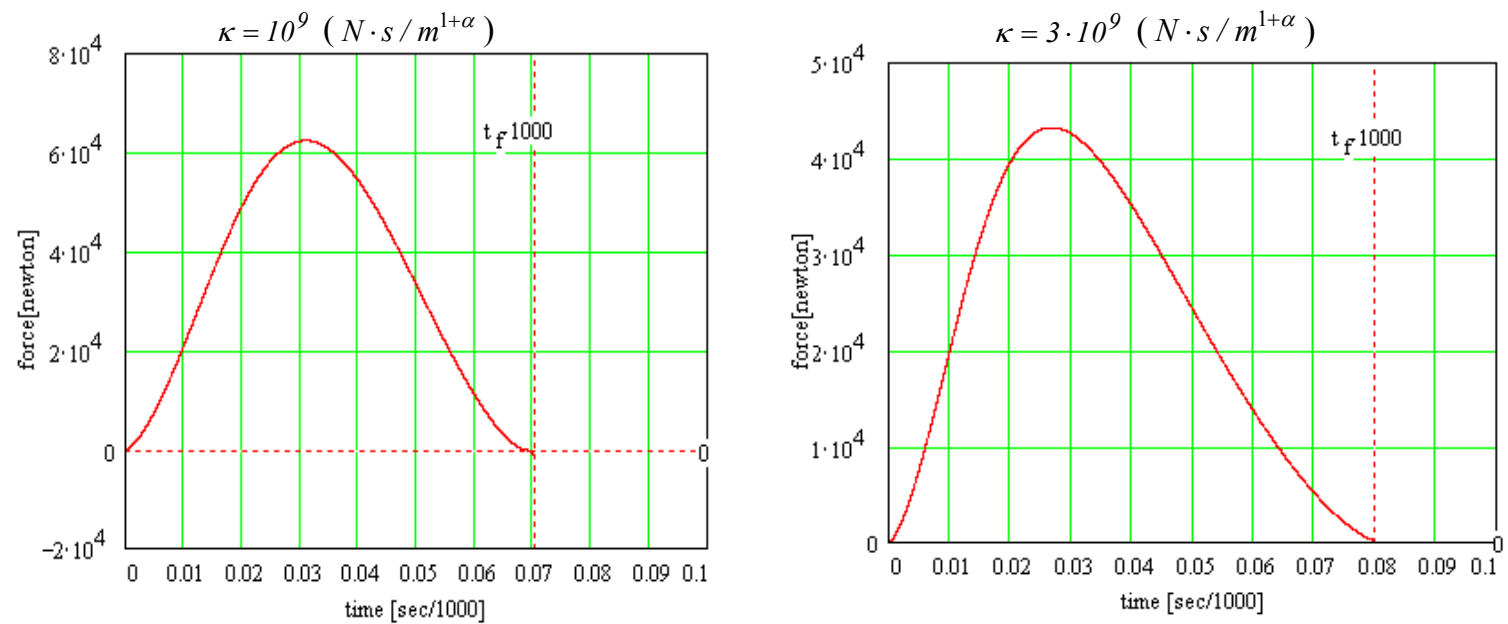

Fig. 7. Impact force variation versus time
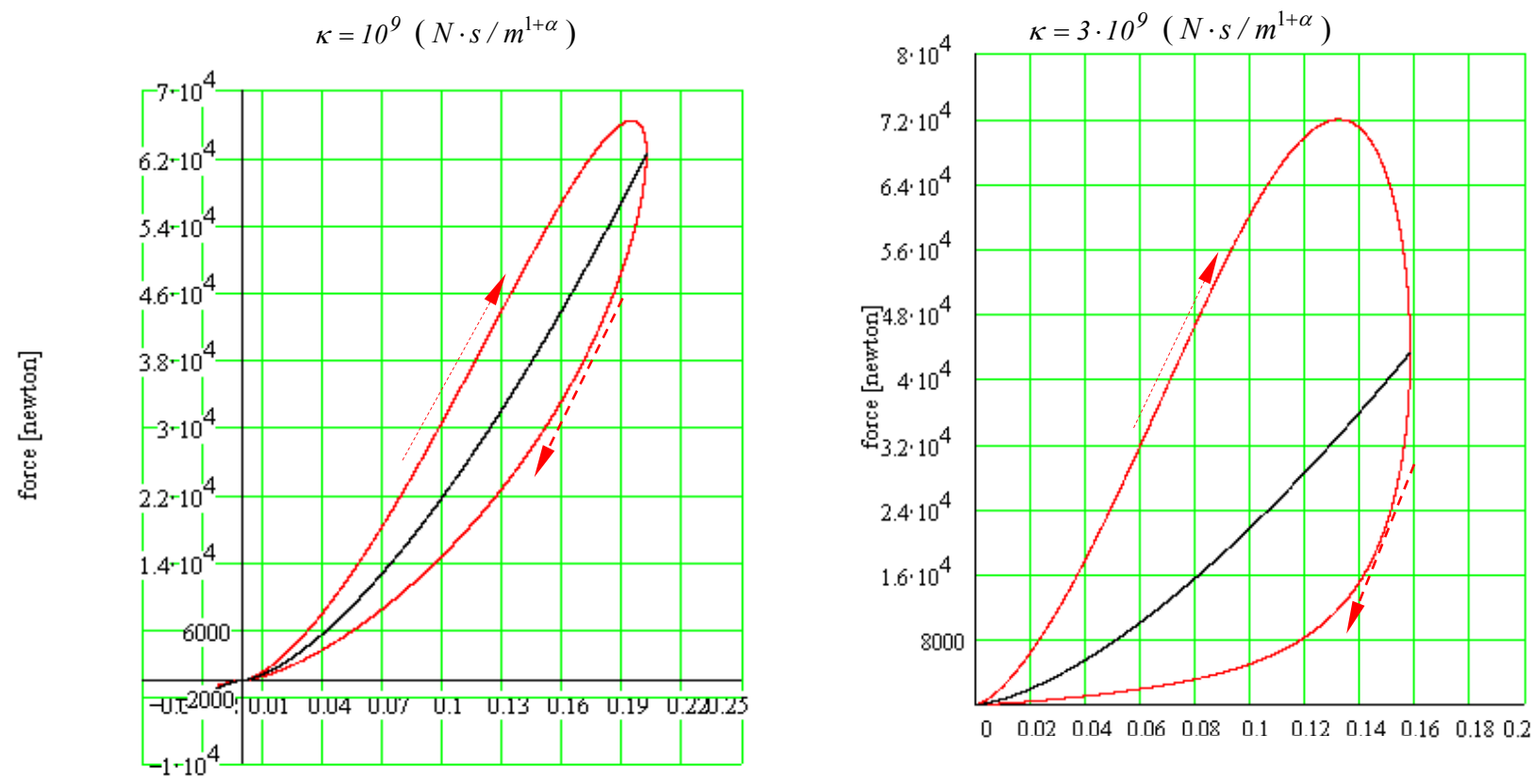

relative deformation $(\mathrm{mm})$

Fig. 8. Hysteresis loops

relative deformation $(\mathrm{mm})$

\footnotetext{
* Corresponding author: florina@fim.usv.ro
} 


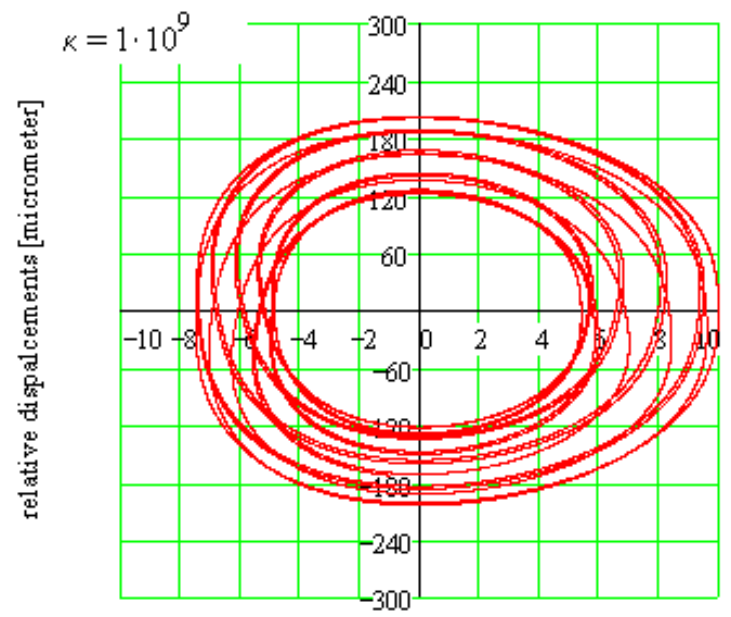

relative velocity $[\mathrm{m} / \mathrm{sec}]$

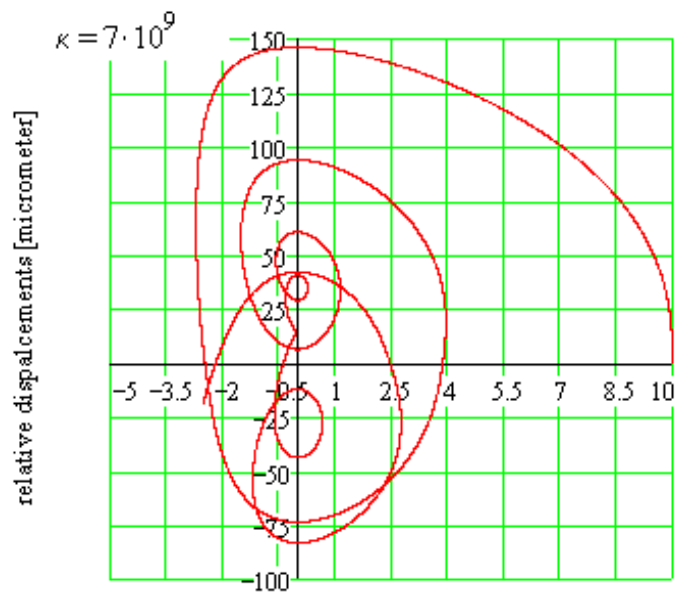

relative velocity $[\mathrm{m} / \mathrm{sec}]$

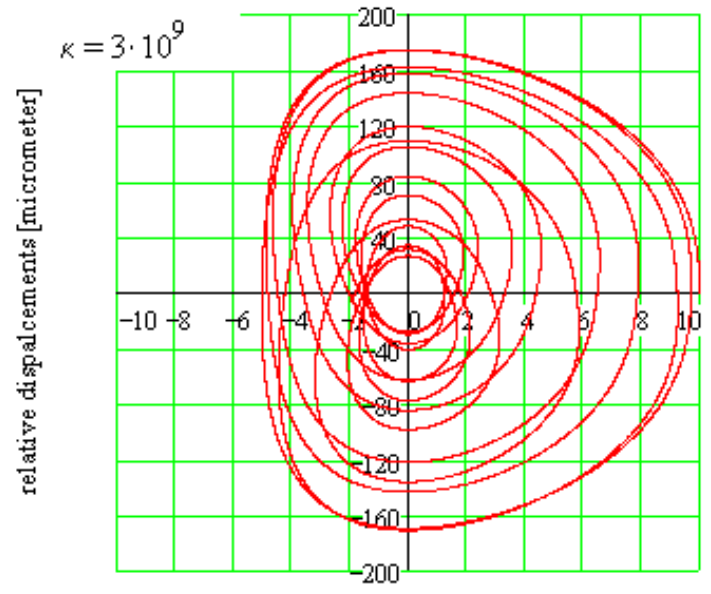

relative velocity $[\mathrm{m} / \mathrm{sec}]$

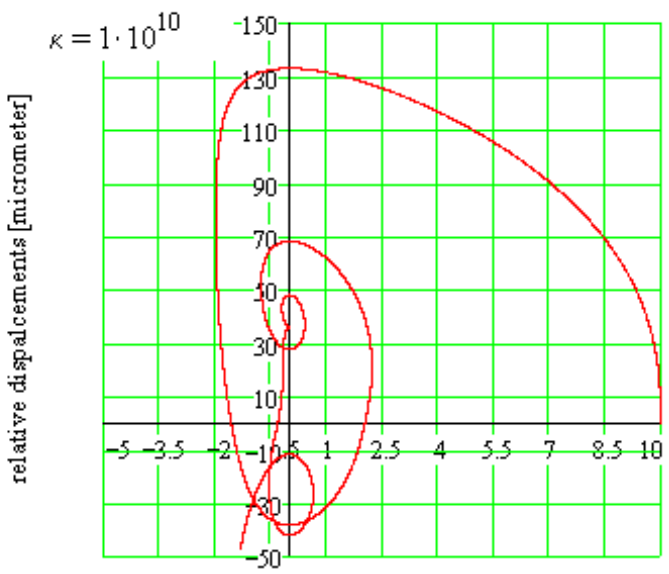

relative velocity $[\mathrm{m} / \mathrm{sec}]$

Fig. 9. Phase diagram for different damping values

A very useful instrument in dynamical analysis of comportment of a system is the phase diagram. In Fig. 9, for the model from Fig. 5, the phase diagrams corresponding for four value of damping are shown.

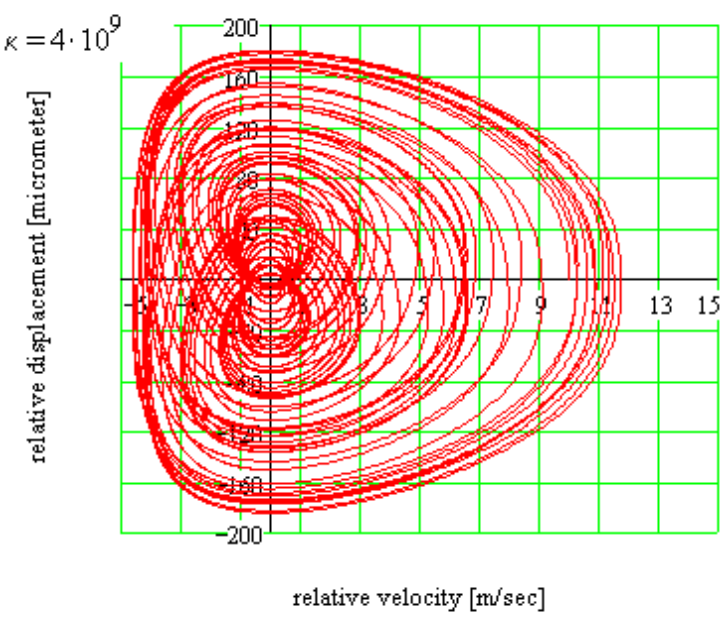

Fig. 10. Phase diagram for critical damping
It can be observed that for lower damping there is only an asymptotic point, while for higher damping values there are two asymptotic points. That means that there is a critical value of damping which splits the two aspects.

In Fig. 10 it is presented the phase diagram for critical damping of the system from Fig. 5.

A number of important conclusions result from Figs. 6-8:

1. The proposed model demonstrates damping with the hysteresis loop closing in origin.

2. The damping work is more important in the approaching phase than during the detaching phase.

3 . The effect of increasing the damping is a slight increase of the time when the displacements of the projectile and target are equal.

4. The area of the hysteresis loop increases significantly when the damping augments.

5. It is observed that the maximum impact force is not in phase with the minimum relative displacement. When the maximum impact force is attained, the minimum relative displacement is delayed with respect to this moment. 
5. Exaggerate augmentation of damping may lead to the increase of maximum impact force.

6. There is an optimum value of damping to which a minimum value of the maximum impact force corresponds.

7. In the phase diagram, the characteristic point describes a selfintersecting path, occurring the possibility that, for higher damping, two asymptotic points exist as indication of bifurcation phenomenon.

\section{Conclusions}

The present paper aims to provide a model for the impact between the sand or dust particles and the surface of the rotor blade of a wind turbine made of composite material. The analysis starts from a model for the impact of composites, broadly applied, and it is highlighted the advantage of this model - the fact that the impact force presents a nonlinear variation, but also the weak point namely the lack of energy dissipation, phenomenon viewed as a fundamental requirement for composite materials. An improved variant of the existent model is proposed in the present work consisting in introducing a nonlinear viscous damper between the projectile and the target made of composite material. Based on recent papers, the damping force is assumed simultaneously proportional to the velocity and to the elastic force. In this manner, the hysteresis loop closed in origin is ensured. The equation of the model is integrated for a few particular cases and the results are presented in graphical form.

This work was supported by a grant of the Romanian Ministry of Research and Innovation, CCCDI UEFISCDI, project number PN-III-P1-1.2-PCCDI-20170404 / 31PCCDI/2018, within PNCDI III”.

\section{References}

1. B. Attaf ed., Advances in Composite Materials Ecodesign and Analysis (InTech Rijeka Croatia 2011)

2. E. Salernitano, C. Migliaresi, J. Appl. Biomater. Biomech. 1(1) 3-18 (2003)

3. Arif Iftekhar, Standard Handbook of Biomedical Engineering and Design 12.1, chapter 12, Biomedical Composites, Downloaded from Digital Engineering Library @ McGraw-Hill (www.digitalengineeringlibrary.com),

(The McGraw-Hill Companies 2004)

4. T. Kondo, Piezoelectric and Acoustic Materials for Transducer Applications Safari A., Akdoğan E.K. (eds) (Springer, Boston, MA 2008)
5. A. C. Baptista, I. Ferreira, J.P. Borges, J. Comp. Biodegr. Polymers, 1, 56-65 (2013)

6. L. Mishnaevsky, Comput. Mech. 50, 195 (2012)

7. S. Abrate, Appl. Mech. Rev. 44 (4), 155 (1991)

8. S. Abrate, Appl. Mech. Rev. 47(11), 517 (1994)

9. S. Abrate, Appl. Mech. Rev. 50 (2), 69, (1997)

10. S. Abrate, Impact on composite structures (Cambridge University Press 1998)

11. S. Abrate, Composite Structures, 51, 129 (2001)

12. L. Mishanevsky, K. Branner, H. Nørgaard Petersen, J. Beauson, M. McGugan, B. F. Sørensen, Materials, 10, 1285 (2017)

13. N. Gauder, J. Phys. Conf. Ser., 524012031 (2014)

14. D. M. Helgren, J. M. Prospero, J. of Climate and Applied Meteorology 26, 9, 1147 (1987)

15. K. L. Johnson, Proc. of the Instit. of Mech. Eng. 196, 363 (1982)

16. R. Olsson, AIAA J, 30(6), 1587 (1992)

17. W. Jordan, P. Smith, Nonlinear differential equation. Problems and Solutions. A Sourcebook for Scientists and Engineers. Oxford Texts in Applied and Engineering Mathematics (Book 11) (Oxford University Pres 2007)

18. W. Gear, Numerical Initial Value Problems in Ordinary Differential Equations (Englewood Cliffs, NJ Prentice-Hall 1971)

19. H. M. Lankarani, P. E. Nikravesh, J. Mech. Design, 112369 (1990)

20. P. Flores, J. Ambrósio, J. C. Pimenta Claro, H. M. Lankarani, Kinematics and Dynamics of Multibody Systems with Imperfect Joints Models and Case Studies (Springer 2011)

21. K. H. Hunt, F. R. E. Crossley, J. Appl. Mech. 7, 440 (1975)

22. S. Dubowsky, F. Freudenstein, 1971, J. of Eng. Ind., B 93(1), 305 (1971)

23. S. Dubowsky, F. Freudenstein, 1971, J. of Eng. Ind., B 93(1), 310 (1971)

24. W. Goldsmith, Impact, The Theory and Physical Behaviour of Colliding Solids (Edward Arnold Ltd, London, England 1960).

25. S. Alaci, F. C. Ciornei, D. Amarandei, C. Filote, D. Cerlinca, S. Spinu, S.: Ann. Oradea Univ. Fasc. Manag. Techn. Eng., X (XX), 1.2102, (2011)

26. S. Alaci, F. C. Ciornei, D. Amarandei, C. Filote, L. Irimescu, S. Spinu, Ann. Oradea Univ. Fasc. Manag. Techn. Eng., X (XX), 2.2221 (2011)

27. S. Alaci, D. A. Cerlincă, F. C. Ciornei, C. Filote, G. Frunză, J. of Physics: Conf. Series 585, 1, 2015. 